Nuclear Instruments and Methods 176 (1980) 349-354

(c) North-Holland Publishing Company

Part IX. Software, simulations, calculations

TRACK-FINDING IN A FINE-GRAINED CALORIMETER

\author{
M. METCALF \\ CERN, Geneva Switzerland
}

In an experiment, whose detector consists, in part, of arrays of large proportional tubes interleaved between marble slabs and surrounded by an iron shell, it is necessary to isolate the trajectories of a small number of muons from the signals of a hadronic shower resulting from a charged-current neutrino interaction. This paper describes the combination of methods used, including a description of the problems of using pulse-height as well as positional information.

\section{A description of the experiment}

A series of experiments is being performed in the neutrino beam of the CERN Super Proton Sychrotron (SPS) by the CERN-Hamburg-AmsterdamRome-Moscow (CHARM) Collaboration, using a detector to investigate charged- and neutral-current interactions of the type:

$$
\begin{aligned}
& \stackrel{(-)}{\nu_{\mu}}+N \rightarrow \stackrel{(+)}{\mu}+X, \\
& \stackrel{(-)}{\nu_{\mu}}+N \rightarrow \stackrel{(-)}{\nu_{\mu}}+X,
\end{aligned}
$$

( $N$ denotes a nucleon, $X$ any final state). The detector has been described in detail in Diddens et al. [1].

\section{The event topologies}

There are a number of different types of event topology produced by the various interactions under study. The principal pattern which has to be analysed is the charged-current event. A clean or ideal chargedcurrent event has the muon clearly separated from the hadronic shower. Unfortunately, we are also confronted with $\pi / \mathrm{K}$ decays inside the shower which give rise to muons, producing an event of the type shown in the figure. A muon may be partially or completely obscured in one projection, and it may undergo some bending, due either to the field in the magnets or to multiple scattering. In the end-calorimeter, the muon can travel through the holes in the proportional tube planes there, so that it is not visible over a significant part of its path. The muons may also undergo bremsstrahlung.
For all these events, we adopt a strategy of working from the back of the detector, where the data are cleaner and try to identify any pattern of signal which corresponds to some minimum definition of a track, outside the shower region, and to match these patterns into tracks in space. The classification of tracks into primary or secondary muons is left to higher-level routines. The emphasis in this high-precision experiment is on full and complete identification of all muons.

\section{The significance of the pulse height}

In the search for muon trajectories it is sensible to make use not only of the positional measurements given by knowing which tubes have fired, but also to endeavour to disentangle the muon from the shower by using the additional information provided by the pulse height registered by each tube. The magnitude of the signal in a given tube is determined by

1) the statistical fluctuations of the Landau distribution;

2) the energy-dependent energy loss;

3) the orientation of the track;

4) the number of traversing particles;

5) space-charge effects.

At the beginning of the experiment it was not realized that this last effect becomes dominant for tracks with a small angle with respect to the wire, and it caused problems not only in early versions of the blanking algorithm described below, but also in the calibration of the response of the tubes, which is performed using cosmic rays in which such tracks are abundant. 


\subsection{The Landau distribution}

The high voltage on the wires of the proportional tubes is adjusted so that the peak of the Landau distribution for $0^{\circ}$ minimum ionizing particles is in channel 10 of the 255 -channel read-out system. This gives a good discrimination for such tracks, combined with a dynamic range sufficient for the measurement of the flux of energy through a tube in the core of most showers. The calibration is carried out by identifying cosmic-ray tracks, which are collected outside machine bursts continuously during data-taking, then correcting them for the track orientation to obtain, finally, the angle-dependent gain constants (see sect. 3.5 below). The cosmic-ray data are then re-read by the progrm to determine the means of the corrected Landau distributions, which are finally calibrated to the scintillators by comparing the visible energy deposited in the two sampling media. For calibration purposes the tubes are treated in groups of 16 , corresponding to one read-out card.

\subsection{Energy loss}

The mean specific ionization shows a steady rise as a function of energy above $1-2 \mathrm{GeV}$. Since in the early stages of the program the energy of a track is unknown, this rise can lead to misinterpretation of a pulse as arising from two low-energy tracks rather than one high-energy one.

\subsection{The track orientation}

The pulse heights' dependence on the track angle is not simply the obvious one of $1 / \cos \theta$. Since the tubes have a square cross-section, the signal can be reduced because the track leaves the tube through the side wall, and if additionally the path length falls below $4 \mathrm{~mm}$, the charge collected drops below an electronic threshold, resulting in the absence of any signal at all. Similarly, there is no signal for tracks at low angles which pass through a plane by the side walls of a pair of tubes. The effect of the side walls is allowed for in the calibration procedure by ignoring all pulses which have a direct neighbour.

\subsection{The particle multiplicity}

The low track multiplicity means that this effect is of relevance only in the analysis of the showers.

\subsection{Space-charge effects}

This effect has been dealt with in Diddens et al. [2] at this conference. Suffice it to summarize here the fact that a particle travelling along a wire sets up a space-charge which changes the gain of the tube in a way which can be described empirically as a strong parabolic shift of the Landau distribution as a function of the angle to the normal to the wire. This implies that the pulse heights seen in one projection are a strong function of the angle in the other. This is taken into account in the calibration procedure by limiting the tracks used to those with an angle below $45^{\circ}$, and by normalizing the tubes to the scintillators for a notional track at $22 \frac{1}{2}^{\circ}$, i.e. for a track at this angle the peak of the Landau distribution is defined arbitrarily to be at $7.3 \mathrm{keV}$, and a global correction to the tube calibration constants is applied such that the sum of the energy measured in the tubes for all the tracks of a run is the same as that seen by the scintillators. This correction factor is about 0.7 .

\section{The blanking procedure}

The track-finding algorithm is preceded by an attempt to blank out those signals which are likely to have originated from the shower development, leaving a much simpler pattern of hits for the subsequent routines. When the first version of the CHARM offline analysis program NAUGHT [3] was written, based on simulated data, the large effect of the space charge (a factor of 6 between $0^{\circ}$ and $22 \frac{1}{2}^{\circ}$ ) was unsus. pected. It was therefore thought possible to remove all signals from the buffer for which the pulse-height lay above a certain threshold, which was set at 3.5 times the peak level for a minimum ionizing particle, thus including most signals from the Landau distribution of a $100 \mathrm{GeV} / c$ particle at $0^{\circ}$, but excluding most signals from the shower region, where many particles traverse each tube, or where very low momentum particles give rise to very large signals. This worked well except for a tendency for tracks to be extended around the edge of a shower, where the blanking had left a low-energy halo. This difficulty was cured by an improvement to the track-following algorithm (as described in sect 5.2 below), combined with the blanking of the direct neighbour of any tube with a pulse above threshold, together with the introduction of a small lower threshold to clear the data of very small signals. 
After the space-charge effect had been understood, and calibrations based upon it produced, it was found that as a result a $0^{\circ}$ track deposited what was apparently only $1 \mathrm{keV}$ of energy, and no correction for this could be applied before the track had been found and its angle determined, i.e. there was no ready means of distinguishing the track of a minimum ionizing particle. At the same time a significant part of the shower went below threshold, which, when re-set at $3.5 \mathrm{keV}$ to compensate, had the effect of blanking one projection of steep tracks. At this stage the blanking procedure had to be re-worked to be based principally on hit patterns rather than on pulse heights; the final version was as follows:

1) On a plane with fewer than three signals, do nothing. This retains signals from clear tracks regardless of their pulse heights.

2) On the other planes, blank out any signal above $3.5 \mathrm{keV}$, unless it has no fired neighbour within four tubes on either side, i.e. if it is an isolated hit. This retains signals from tracks at the side of or just penetrating a shower. If the signal is removed, its direct neighbours are also taken out, and if it has direct neighbours, their neighbours are also removed, thus effectively removing contiguously firing tubes from showers, whilst retaining hits from close tracks. In the magnet system the threshold is set higher as there is less risk of contamination by shower hits, and the track-finding algorithm used there is more sensitive to missing points.

3) On the last four planes of the detector, where there is a double measurement of the muon trajectories, each signal is required additionally to have a corresponding signal in the other plane of the same projection, unless this results in all signals being removed.

4) To deal with the problem of tracks which go through the central holes in the end-calorimeter, signals corresponding to the hole positions are generated if the end-calorimeter has very few signals in total but the end-magnets have a number consistent with the presence of a track there.

\section{The track finding}

The track-finding operates on the hits remaining after the blanking procedure, the hits being stored as bit patterns using several words per plane and accessed by fast machine language functions. It begins by trying first to identify tracks in the magnet system where conditions are cleanest, and where matching information is available. Any tracks found there are followed back into the target calorimeter, where a concurrent search for other tracks is made. In order to achieve the aim of identification of all muons, the minimum track length in the target calorimeter is set to less than half that required to distinguish a muon from a pion, namely four points in each projection (or five in just one) rather than 10 .

\subsection{The combinatorial search in the magnet system}

Taking the last plane of the target calorimeter to belong to the set of planes in the main magnet system, we see that there are eight non-inclined planes in each of the two projections.

The basic assumption is made that the particle trajectories in the magnet system can be adequately represented by parabolas, and the method used in the search for their tracks follows from this.

The steps in the search are the following:

1) Set up, in the first projection, arrays containing the coordinates of all the corresponding fired orthogonal tubes in the magnet system (planes 78-96). In the case of the last four planes, only one plane of each projection is used (table 1 ).

2) A choice is then made of the first, middle, and last pivot planes; that is, planes whose fired tubes are used to define the coefficients of parabolas which are then used in the search for additional points on the other planes. This choice is made following table 1 , taking the first time through only combinations 1,2 , and 5 (the numbers in parentheses indicate planes used if the first-choice plane is empty).

Table 1

Pivot planes selection

\begin{tabular}{|c|c|c|c|c|}
\hline Combination & First & Middle & Last & $\begin{array}{l}\text { Minimum no. } \\
\text { of extra hits }\end{array}$ \\
\hline 1 & 1 & $5(4,6)$ & 7 & 2 \\
\hline 2 & 1 & $4(3)$ & 6 & 1 \\
\hline 3 & 1 & $4(3)$ & 5 & 1 \\
\hline 4 & 1 & 3 & 4 & 1 \\
\hline 5 & 2 & $5(4,6)$ & 7 & 1 \\
\hline 6 & 2 & $5(4)$ & 6 & 1 \\
\hline 7 & 2 & 4 & 5 & 1 \\
\hline 8 & 3 & $5(4)$ & 7 & 1 \\
\hline 9 & 3 & 5 & 6 & 1 \\
\hline 10 & 1 & $3(2)$ & 4 & 0 \\
\hline 11 & 1 & 2 & 3 & 0 \\
\hline 12 & 2 & 3 & 4 & 0 \\
\hline
\end{tabular}




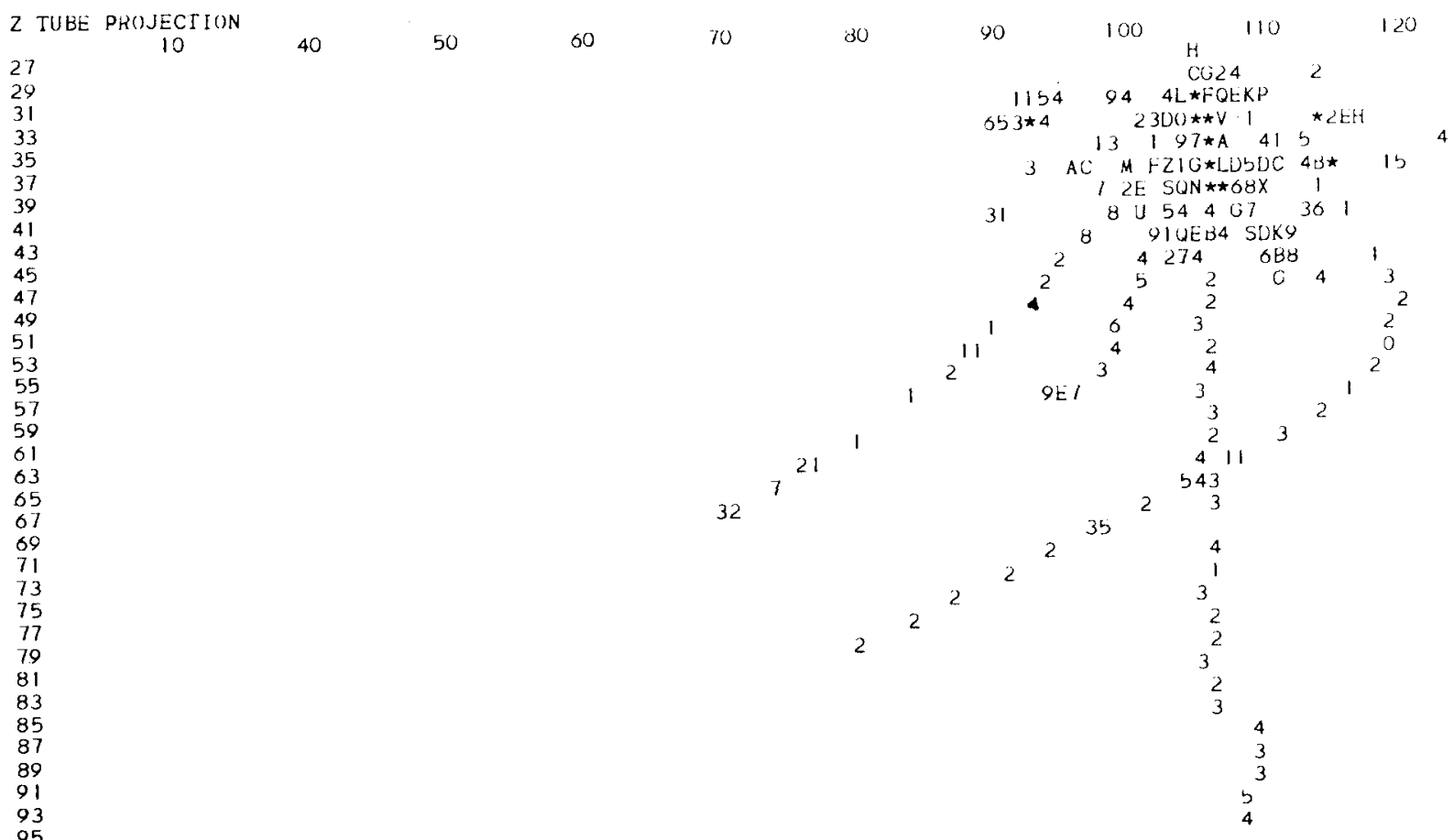

Fig. 1. A multi-muon event. The numbers across the top of the page are tube numbers, those down the side are plane numbers. Each fired tube is represented by its pulse-height on a scale from 0 to $35 \mathrm{keV}(0 \mathrm{to} \mathrm{Z})$, the symbol * standing for a signal above $35 \mathrm{keV}$. We note four muons, the longest passing through the whole detector, the second longest being focussed back by the frame magnets to cross the longest, and the two shortest showing signs of multiple scattering.

3) For each combination of points of the first and last pivot planes, a new coordinate system is defined whose abscissa is parallel to the straight line joining them. The points on the middle pivot plane are taken successively and used to define the coefficients of parabolas in the rotated system. A check on the curvature is made.

4) A search is next made over all points on all nonpivot planes lying between the first and the last pivot planes. The closest point lying within a certain tolerance to the prediction on the plane in question is added to the track; points which are assigned to a track are deleted from the coordinate array. If the track is finally rejected because it has too few points for the combination (see the table), the points are restored.

5) The procedure is performed a second time, now for all the combinations of table 1 and with wider limits.

6) The whole procedure is repeated for the second projection.

7) A sub-routine is called to match tracks found in the projections into tracks in space using the informa- tion from the inclined planes. On each of the three planes the quantity

$u=y \cdot \cos \alpha+z \cdot \sin \alpha, \quad \alpha=12^{\circ}$

(or its equivalent for $v$ ) is calculated for each combination of tracks, and a search made for a corresponding signal on the plane. Tracks for which this condition is fulfilled on a minimum number of planes and which are of similar length are then matched in space. Owing to the significant geometrical inefficiency of the tubes, the minimum number of hits required has been set to zero in cases where only one track has been found in each projection; in all other cases it is two.

\subsection{Track-following in the target calorimeter}

The track-following in the target calorimeter is performed mainly by a single large routine for reasons of efficiency. It receives as input a copy of the tube firing patterns, a list of track sections already found in the magnet system, and the detector constants.

It works progressively through the calorimeter 
plane by plane (but taking each projection in turn) starting at the last plane and finishing at the first one containing signals. On each plane it unpacks the tube firing paitern and then makes a series of tests:

1) Can any track which has been found in the magnet system be continued by the addition of a point from the firing pattern of this plane? If this is the case, the point is added to the track and removed from the list of fired tubes.

2) Can any track which has been found to terminate in the target calorimeter be continued by the addition of a point remaining on this plane? If this is the case the point is added to the track and removed from the list of fired tubes.

3) Can any point still remaining be used to form the end of a track stopping in the target calorimeter or leaving the sides? To make this test the points on the two preceding (i.e. upstream) planes are unpacked, and a search for combinations of three points fulfilling a straight-line condition is made. All such combinations are stored in a dynamic buffer and marked as being candidates for continuation after two further steps in procedure.

After this attempt to use all the points on the plane, a check is made on all the tracks which have not been continued. In the case of magnet tracks which have not been continued for a certain number of planes, the tracks are marked as being complete and no further attempt will be made to continue them. In the case of tracks stopping in the target calorimeter they are similarly marked, unless they have too few points, when they are deleted.

When attempting to continue tracks backwards in the manner described above, the last point added to the track together with the second to last point are used to predict a point on the plane being examined. If an actual point lies sufficiently close to this predicted point and if the resultant change in slope of the track is not too large, then the acceptance conditions are fulfilled and the point is used. The new value of the local track angle is stored for use in the next step.

When the pass through the calorimeter for a given projection is complete, all tracks terminating in the target calorimeter are fitted to straight lines and a chisquare estimator calculated. Those tracks which do not fulfil the straight-line condition at the $0.1 \%$ level are passed to a routine to have their first few points examined and possibly removed. This is not done if an explicit search for non-straight tracks is being made, i.e. stopping muons, but for other tracks there are two main reasons why a track may not fulfill the straight-line test:

1) the track is truly curved, because it is in the iron or has undergone scattering;

2) the track has been wrongly followed in the shower region.

For this second case, the routine tries to improve the track by imposing on its start the same condition as was imposed on its end, namely three points on three consecutive planes. If this condition is not fulfilled, points are removed until it is.

The tracks in the projection are next sorted into an order determined by their goodness of fit.

After the tracks in both projections have been found, they are passed to another routine for final matching in space, and most spurious point combinations are eliminated at this stage. The problem of matching tracks in space with no inclined information available is approached by making use of the various characteristics of the tracks in the two projections. In this routine, the tracks are first matched on the basis of their total length and the position of their midpoints, taking them in an order determined by their goodness of fit to a straight line, and imposing in the first of two passes the requirement of a common end plane. For tracks which are heavily obscured by a shower in one projection and virtually complete in the other, this method does not work and so any tracks remaining are matched solely on the basis of their end points. Any sufficiently long tracks then remaining are designated as one-projection tracks.

Finally, straight-line fits and possible point removal are performed on those tracks which were continued from the manget system.

\subsection{The final stage}

The blanking procedure can cause the loss of points along a track, either because of a large pulseheight or because a section of track was too close to part of the shower. To restore points which are lost in this way, a routine makes a pass along the track trying to add any missing points, using straight-line interpolations. At the end of a track an attempt is made to extrapolate it, this being especially important for tracks with straggled ends, or for those which are stopped in or leave the end-calorimeter without giving a sufficient number of hits for a magnet track to have been found.

As a last step, tracks which have been found in two sections, mainly due to intense bremsstrahlung 
somewhere on the track, are joined into one, on the basis of their straight-line parameters and positions in the detector.

\section{Conclusion}

It has been found possible to develop a method of finding muons in a fine-grained calorimeter with an efficiency of more than $99 \%$ for true muon tracks. An initial attempt to use the pulse-height information for cleaning the data prior to the track-finding has had to give way to one incorporating pattern information as well, owing to space-charge effects. The final quality of the tracks from the point of view of the resolution of the left-right ambiguity is impaired by tube inefficiencies, and by an attempt to use the whole calorimeter for shower development, thereby causing many muons to be seen in only part of the magnet system (see also ref. 4).

This work is part of the CERN-Hamburg-Amsterdam-Rome-Moscow Collaboration and owes much of its success to the many contributions from its members.

\section{References}

[1] A.N. Diddens et al. (CHARM Collaboration), Nucl. Instr. and Meth. (to be published, 1980).

[2] Idem (CHARM Collaboration), these proceedings, p. 189.

[3] J. Allaby, ed., NAUGHT users manual, CHARM internal document, CERN (1980).

[4] K.M. Mess, M. Metcalf, R. Orr, B. Kröger and A. Rosanov, CERN-DD/80-7 (1980). 